\title{
System-Level Coupled Modeling of Piezoelectric Vibration Energy Harvesting Systems by Joint Finite Element and Circuit Analysis
}

\author{
Congcong Cheng, ${ }^{1}$ Zhongsheng Chen, ${ }^{1,2}$ Hongwu Shi, ${ }^{1}$ Ziniu Liu, ${ }^{1}$ and Yeping Xiong ${ }^{2}$ \\ ${ }^{1}$ Science and Technology on Integrated Logistics Support Laboratory, National University of Defense Technology, \\ Changsha 410073, China \\ ${ }^{2}$ Faculty of Engineering and the Environment, University of Southampton, Boldrewood Campus, Southampton SO16 7QF, UK
}

Correspondence should be addressed to Zhongsheng Chen; chenzhongsheng@nudt.edu.cn

Received 10 December 2015; Revised 27 January 2016; Accepted 3 February 2016

Academic Editor: Lorenzo Dozio

Copyright (C) 2016 Congcong Cheng et al. This is an open access article distributed under the Creative Commons Attribution License, which permits unrestricted use, distribution, and reproduction in any medium, provided the original work is properly cited.

A practical piezoelectric vibration energy harvesting (PVEH) system is usually composed of two coupled parts: a harvesting structure and an interface circuit. Thus, it is much necessary to build system-level coupled models for analyzing PVEH systems, so that the whole PVEH system can be optimized to obtain a high overall efficiency. In this paper, two classes of coupled models are proposed by joint finite element and circuit analysis. The first one is to integrate the equivalent circuit model of the harvesting structure with the interface circuit and the second one is to integrate the equivalent electrical impedance of the interface circuit into the finite element model of the harvesting structure. Then equivalent circuit model parameters of the harvesting structure are estimated by finite element analysis and the equivalent electrical impedance of the interface circuit is derived by circuit analysis. In the end, simulations are done to validate and compare the proposed two classes of system-level coupled models. The results demonstrate that harvested powers from the two classes of coupled models approximate to theoretic values. Thus, the proposed coupled models can be used for system-level optimizations in engineering applications.

\section{Introduction}

Nowadays, low power consumption wireless sensor networks (WSNs) are widely used in military and industrial fields, such as equipment operation condition monitoring, bridge health monitoring, and intelligent transportation. As for most applications, WSNs are far from power lines. In particular, wireless sensor nodes may need to be embedded into mechanical structures. Under those cases, it is difficult to power WSNs by wires, so that battery is the most conventional solution for WSNs. However, batteries need to be replaced regularly due to their limited life spans. For WSNs placed in highly dangerous or unreachable areas, it is hard and even impossible to replace batteries. Nowadays, the most suitable solution to extend the life of a WSN is to harvest environmental energy to generate electrical energy. Up to now, many efforts have been made on harvesting energy from environmental vibrations, such as mechanical vibrations cause by operating equipment or in-service bridges. Generally speaking, vibration energy harvesting can be carried out by electrostatic [1], electromagnetic [2], or piezoelectric mechanisms [3, 4]. In particular, piezoelectric mechanism is of particular interest due to high energy density and electromechanical coupling coefficient. Thus, piezoelectric vibration energy harvesting (PVEH) has been widely studied to realize self-powered WSNs $[5,6]$.

A typical PVEH system usually can be divided into two parts. The first part is the harvesting structure composed of piezoelectric material, elastic base, and electrodes, which determines the electrical energy transformed from the vibration energy. The second part is the electrical part composed of the interface circuit and storage unit, which determines the efficiency of the electrical energy from the harvesting structure into the storage unit such as capacitor. By now, many works have been conducted on those two parts. For the harvesting structure, piezoelectric cantilever beams are widely used due to their tremendous application potential, 


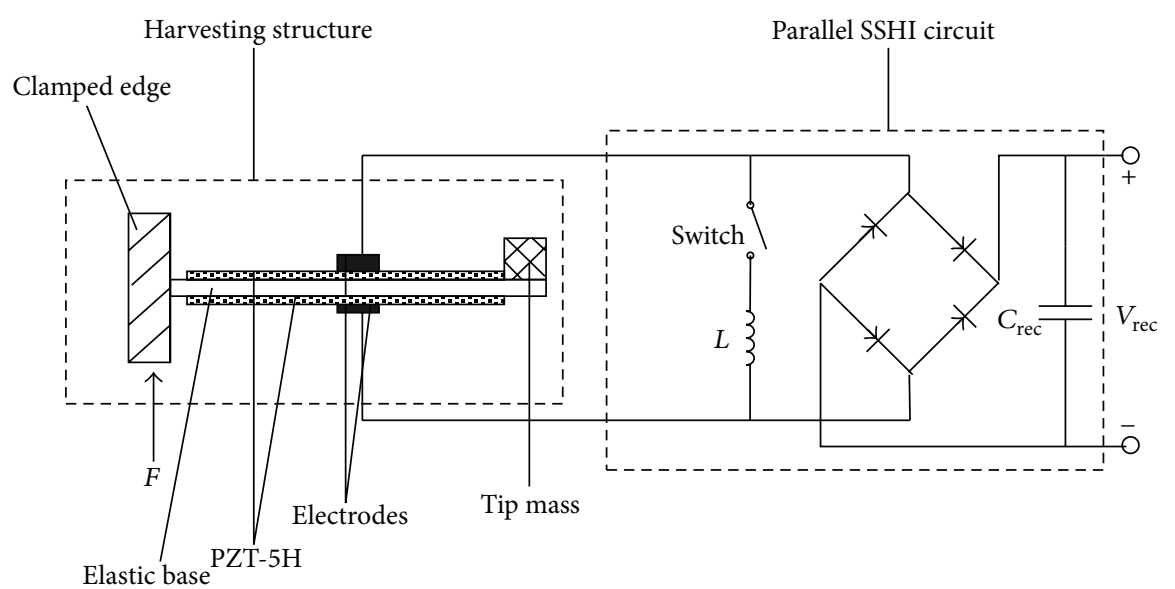

FIGURE 1: Structure of a PVEH system with a parallel SSHI circuit.

including unimorph and bimorph type beams. For the interface circuit, standard energy harvesting (SEH) interface circuit composed of a full-wave bridge rectifier is first used. But its efficiency is very low because intrinsic capacitance of a piezoelectric patch is often very large and varies with exciting frequencies, so that it is very difficult for SEH circuits to achieve optimal impedance matching [7]. In order to overcome this drawback, a technique called synchronized switch harvesting on inductor (SSHI) has been proposed to enhance the power output of a PVEH system, including parallel SSHI (P-SSHI) and series SSHI (S-SSHI) circuits [8]. SSHI interface circuits are nonlinear, so it has been testified that SSHI-based circuits can increase the harvested power by several times compared to that of a SEH circuit under the same inputs.

It can be seen that most existing works focus on either the harvesting structure or the interface circuit. In particular, many works on circuits in literature make the excessively simplifying assumption of a purely capacitive model of the piezoelectric circuit. In practice, however, the harvesting structure and the interface circuit are coupled due to inverse piezoelectric effect. Dynamic behaviors of the harvesting structure are affected by the interface circuit. Conversely, the output voltage of the harvesting structure will affect the performance of the interface circuit. From the viewpoint of engineering applications, the overall efficiency of a PVEH system is truly expected, which depends on many contributing factors such as the vibration excitations, the geometries and materials of the harvester structure, and the interface circuit. For this reason, a PVEH system should be considered as a whole for optimization to obtain a high overall efficiency. Thus, it is much necessary to build a system-level coupled model and carry out coupled analysis. Nowadays, one widely used coupled model for a PVEH device is the equivalent electromechanical circuit defined by lumped parameters involving both mechanical and electrical quantities [9-12]. And then a two-port representation can reasonably account for all feedbacks between the mechanical and the electrical parts. Its main difficulty is to estimate rapidly and accurately all model parameters, while direct measurements are timeconsuming. In recent years, finite element modeling methods are introduced into the field of PVEH, but it is difficult to directly combine finite element and circuit analysis due to the nonlinearity of interface circuits. In [13] finite element solvers are coupled to SPICE circuit software to simulate electrical responses of a PVEH device. However, it requires transferring data between the finite element solver and SPICE simulator at each iteration, which leads to low calculating efficiency. Hence, there is an urgent need to rapidly and accurately establish a system-level coupled model to analyze and optimize a PVEH system.

In this paper, a typical PVEH system including a bimorph piezoelectric harvesting structure and a parallel SSHI circuit is taken into account. Then joint finite element and circuit analysis is combined to construct system-level coupled models of the PVEH system. The innovation of this paper is to propose two classes of coupled models. The first one is a system-level coupled circuit model, which can be independently built in circuit simulation software. The second one is a system-level coupled finite element model, which can be independently built in finite element software. Both models do not require data transferring during working and experimental tests for parameters estimations. The remainder of this paper is organized as follows: current problems of coupled modeling methods of a typical PVEH system are summarized in Section 2. In Section 3, coupled modeling methodology of the PVEH system is demonstrated based on joint finite element and circuit analysis and two classes of coupled models are proposed. Then the first-class model is investigated in Section 4 and the second-class model is investigated in Section 5. Simulations and experiments are done in Section 6. Finally, Section 7 concludes the whole paper.

\section{Problem Statements}

In this paper, a typical PVEH system composed of a bimorph cantilever beam and a parallel SSHI circuit is shown in Figure 1. In order to integrate the harvesting structure with the interface circuit, the most conventional way is to build the equivalent circuit model of the harvesting structure. Firstly, the harvesting structure can be modeled as an equivalent 


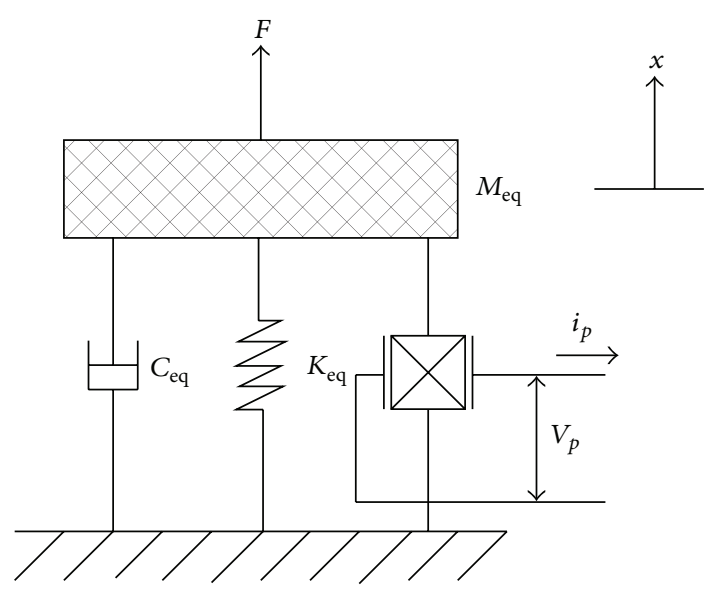

FIGURE 2: Equivalent mechanical model of the harvesting structure.

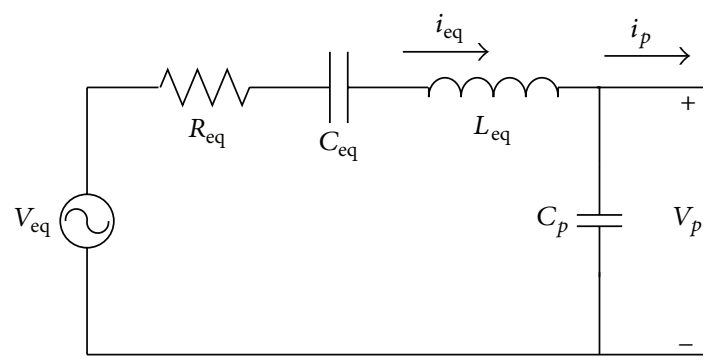

FIgURE 3: Equivalent circuit model of the harvesting structure.

mechanical model involving mass $\left(M_{\mathrm{eq}}\right)$, damping $\left(\eta_{\mathrm{eq}}\right)$, spring $\left(K_{\mathrm{eq}}\right)$, and piezoelectric unit as shown in Figure 2.

Furthermore, the harvesting structure can be equivalent to an electrical current source [14]. In this case, the above equivalent mechanical model can be transformed into an equivalent circuit model shown in Figure 3 by using analogies between mechanical and electrical quantities. $V_{\text {eq }}, I_{\text {eq }}$ are the equivalent voltage and current, respectively. The equivalent inductor $L_{\text {eq }}$ is given by the equivalent mass $M_{\text {eq. }}$. The equivalent capacitor $C_{\mathrm{eq}}$ is given by the equivalent stiffness $K_{\mathrm{eq}}$. The equivalent resistor $R_{\mathrm{eq}}$ is given by the equivalent damping ratio $\eta_{\text {eq }} \cdot C_{p}$ is the parasitic capacitor of the piezoelectric material. Furthermore, we will have

$$
\begin{aligned}
V_{\mathrm{eq}} & =-\frac{M \ddot{x}}{\alpha}, \\
i_{\mathrm{eq}}(t) & =\alpha \dot{x}, \\
L_{\mathrm{eq}} & =\frac{M_{\mathrm{eq}}}{\alpha^{2}}, \\
R_{\mathrm{eq}} & =\frac{\eta_{\mathrm{eq}}}{\alpha^{2}}, \\
C_{\mathrm{eq}} & =\frac{\alpha^{2}}{K_{\mathrm{eq}}},
\end{aligned}
$$

where $\alpha$ is the force-voltage coupling factor.
However, if geometric shape of the harvesting structure is irregular, (1) will be less accurate. Finally, the equivalent circuit model can be directly connected to the interface circuit. By this way, coupled analysis of the PVEH system can be carried out. However, now three main difficulties still coexist in this task: (a) accurate identification of equivalent model parameters is always time-consuming based on experimental tests. In particular, an exact value of the equivalent mass $M_{\mathrm{eq}}$ cannot be easily determined by direct measurements [15]; (b) all geometric and material parameters of the harvesting structure will not appear in this coupled model, so it is difficult to optimize the harvesting structure itself; (c) the parallel SSHI circuit is nonlinear, instead of a linear one. Thus, a rapid and reliable modeling method of coupled behaviors between the harvesting structure and interface circuit is highly desirable.

\section{Coupled Modeling Methodology of the PVEH System by Joint Finite Element and Circuit Analysis}

In order to overcome the above difficulties in calculating equivalent model parameters, finite element analysis (FEA) is introduced for coupled modeling of the PVEH system in this paper. Nowadays, as we all know, many commercial FEA software can be used for structural and circuit analysis, such as ANSYS and COMSOL. Based on it, two classes of systemlevel coupled modeling methods are proposed as follows.

The first-class model is to integrate the equivalent circuit model of the harvesting structure with the interface circuit as shown in Figure 4(a). Its basic idea is to transform the harvesting structure into an equivalent circuit, which has been stated in Section 2. Different from previous works [15], here finite element model of the harvesting structure is built and its equivalent model parameters are estimated by FEA. Then a system-level coupling electrical model is built, which can be analyzed in circuit analysis software. Advantages of this method include the following: (a) arbitrary-shaped harvesting structures can be analyzed; (b) it is more rapid and accurate to estimate all model parameters than by direct measurements.

The second-class model is to integrate the equivalent electrical impedance of the interface circuit into the finite element model of the harvesting structure as shown in Figure 4(b). Its basic idea is to directly build the interface circuit in finite element software. However, SSHI circuits include nonlinear components, such as diodes and transistors. While most commercial FEA software can only simulate a linear circuit, it is difficult to directly build nonlinear SSHI circuits by FEA software. In order to solve this problem, equivalent electrical impedance of the SSHI circuit can be derived based on circuit theories. By this way, the nonlinear SSHI circuit can be represented by linear components. Then the SSHI circuit can be directly connected to the harvesting structure in FEA software, so that a system-level coupled model can be built and analyzed. Advantages of this method include the following: (a) all geometric and material parameters of the harvesting structure are retained so that the harvesting 


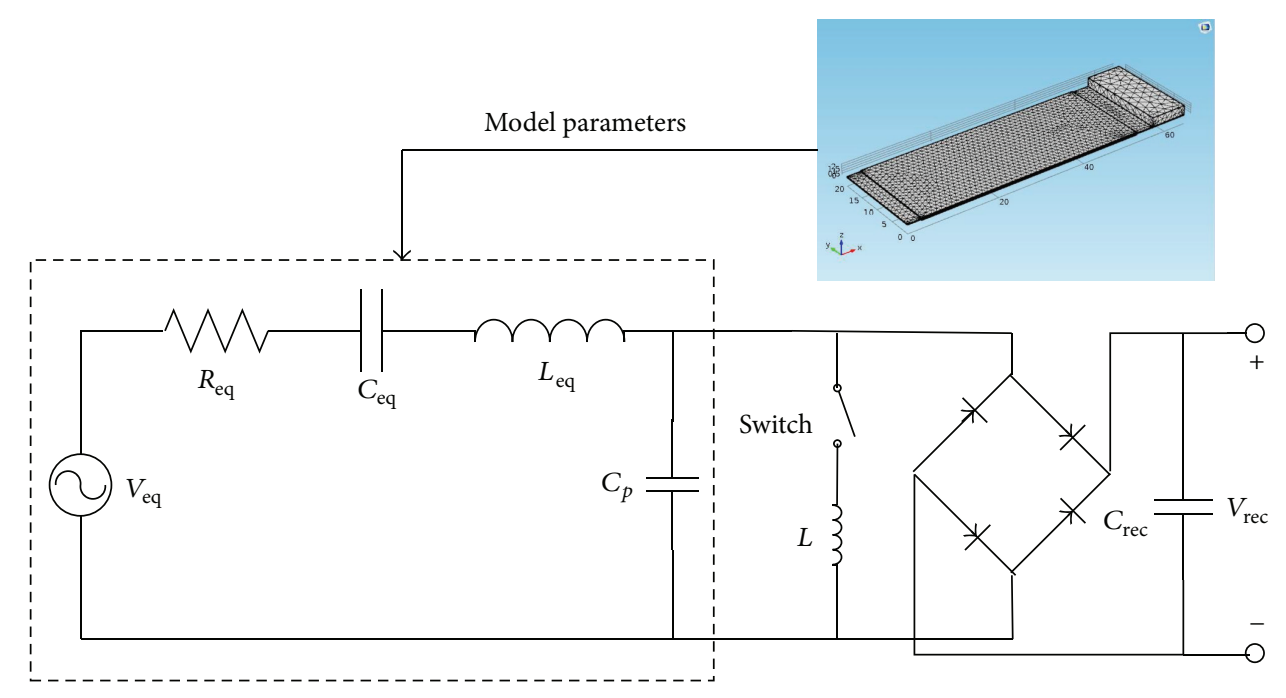

(a) A system-level coupled circuit model

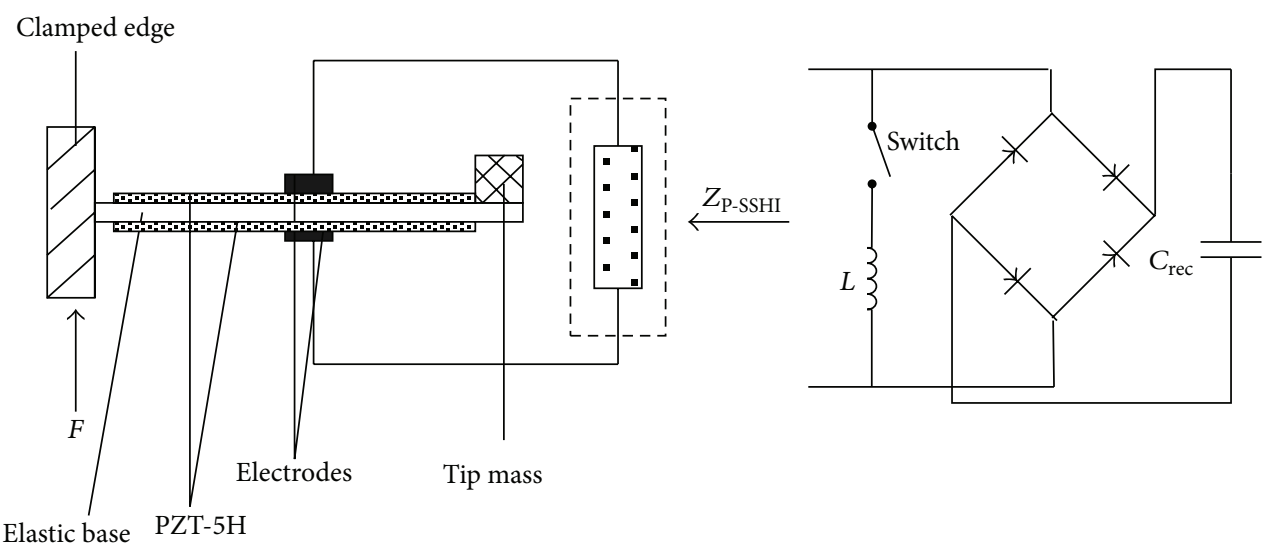

(b) A system-level coupled finite element model

FIGURE 4: Schematic diagrams of the proposed two classes of coupled models.

structure can be optimized accounting for the effects of the interface circuit; (b) no circuit software is needed.

\section{The First-Class Coupled Model: Estimation of Equivalent Model Parameters by FEA}

As for the first-class coupled model in Figure 4(a), the key step is to accurately estimate equivalent model parameters by finite element analysis. Here, a bimorph piezoelectric harvesting structure is taken into account and its finite element model is built by the COMSOL Multiphysics software, as shown in Figure 5.

For the sake of estimating equivalent model parameters, a simple four-step method is presented as follows.

Step 1 (calculation of short-circuit resonant frequency $\left(f_{\text {sc }}\right)$ ). The output terminal of the harvesting structure is connected to the ground, so that a short-circuit condition is formed. Then "eigenfrequency analysis" is conducted in COMSOL and the short-circuit resonant frequency $f_{\text {sc }}$ can be calculated. On the other hand, the formula of $f_{\mathrm{sc}}$ can be described as in (2) according to Figure 3:

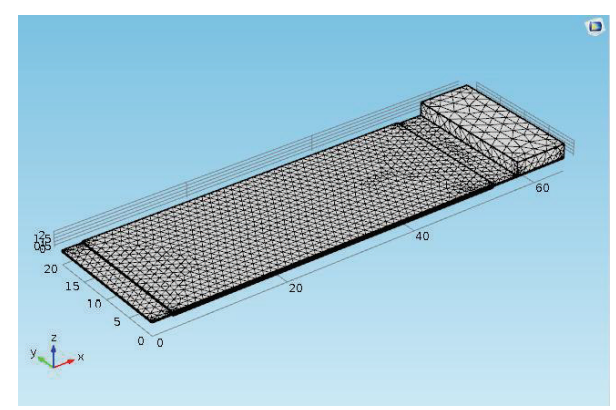

FIGURE 5: Finite element model of the harvesting structure by COMSOL software.

$$
f_{\mathrm{sc}}=\frac{1}{2 \pi} \sqrt{\frac{1}{L_{\mathrm{eq}} C_{\mathrm{eq}}}} .
$$

Step 2 (calculation of open-circuit resonant frequency $\left(f_{\text {oc }}\right)$ ). No load is connected to the harvesting structure so that an open-circuit condition is formed. Then "eigenfrequency analysis" is conducted in COMSOL and the open-circuit 


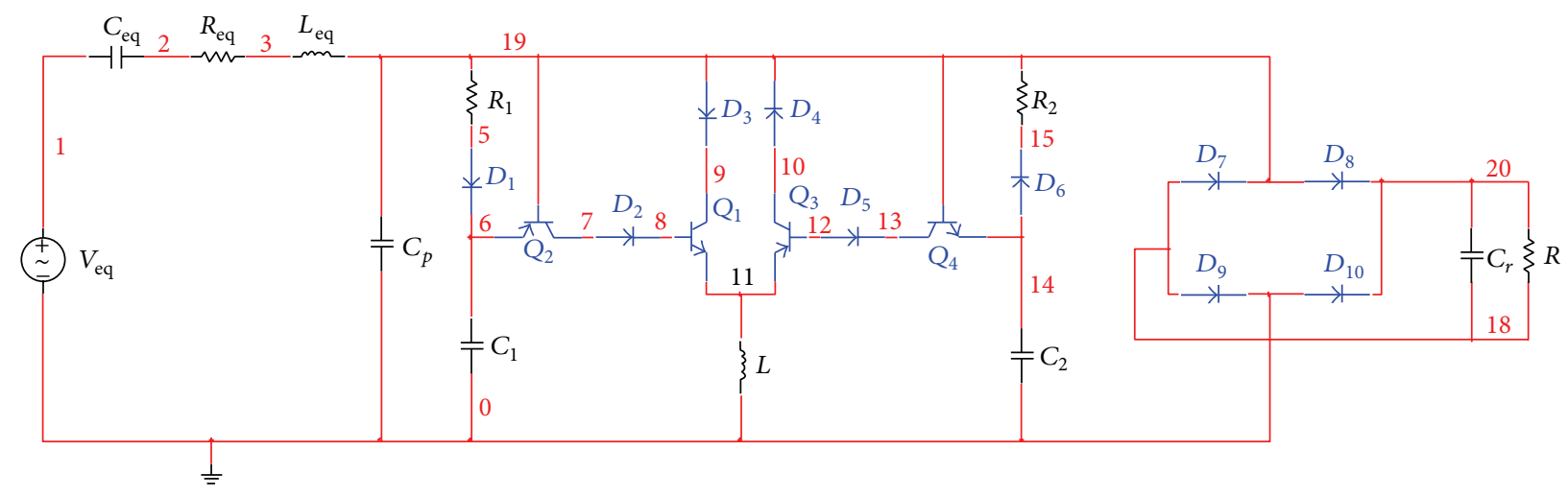

FIGURE 6: The first-class system-level coupled model of the PVEH system.

resonant frequency $f_{\text {oc }}$ can be calculated. On the other hand, the formula of $f_{\text {oc }}$ can be described as in (3) according to Figure 3:

$$
f_{\mathrm{oc}}=\frac{1}{2 \pi} \sqrt{\frac{C_{\mathrm{eq}}+C_{p}}{L_{\mathrm{eq}} C_{\mathrm{eq}} C_{p}}} .
$$

Step 3 (calculation of output charges under a given DC voltage). Under the open-circuit condition, an arbitrary DC voltage $V_{0}$ is functioned across the two electrodes on the harvesting structure. Then the corresponding output charge $Q_{0}$ is calculated. On the other hand, the formula of $Q_{0}$ can be described as in (4) according to Figure 3:

$$
Q_{0}=\left(C_{p}+C_{\mathrm{eq}}\right) V_{0}
$$

Step 4 (calculation of open-circuit voltages under two given harmonic excitations). Under open-circuit conditions, two harmonic excitations $\left(F_{1}=f_{1} \cos \omega_{1} t, F_{2}=f_{2} \cos \omega_{2} t\right)$ are exerted on the free end of the harvesting structure, respectively. Then the corresponding open-circuit voltage amplitudes, $V_{\mathrm{oc}}^{1}, V_{\mathrm{oc}}^{2}$, are calculated. On the other hand, the formulas of $V_{o c}^{1}, V_{o c}^{2}$ can be described as in (5a) and (5b) according to Figure 3:

$$
\begin{aligned}
& V_{\mathrm{eq}}=\frac{\left|R_{\mathrm{eq}}+j\left(\omega_{1} L_{\mathrm{eq}}-1 / \omega_{1} C_{\mathrm{eq}}-1 / \omega_{1} C_{p}\right)\right|}{\left|j / \omega_{1} C_{p}\right|} V_{\mathrm{oc}}^{1} \\
& V_{\mathrm{eq}}=\frac{\left|R_{\mathrm{eq}}+j\left(\omega_{2} L_{\mathrm{eq}}-1 / \omega_{2} C_{\mathrm{eq}}-1 / \omega_{2} C_{p}\right)\right|}{\left|j / \omega_{2} C_{p}\right|} V_{\mathrm{oc}}^{2} .
\end{aligned}
$$

By now, model parameters $L_{\text {eq }}, C_{\text {eq }}$, and $C_{p}$ can be estimated from (2) (4) and $V_{\text {eq }}, R_{\text {eq }}$ can be estimated from (5a) and (5b). Finally, the first-class coupled model can be alone built in the Multisim software by connecting the equivalent model to the parallel SSHI interface circuit, as shown in Figure 6. Here, the parallel SSHI circuit is composed of a bridge rectifier and a self-powered switch circuit [16]. The forward voltage drop of each diode is denoted as $V_{D}$ and a pure resistor $R$ is utilized as the load.

\section{The Second-Class Coupled Model: Calculation of the Equivalent Electrical Impedance by Circuit Analysis}

As for the second-class coupled model in Figure 4(b), the key principle is to transform the parallel SSHI circuit into the corresponding equivalent electrical impedance. Liang and Liao [15] have proposed an effective way of impedance modeling of different interface circuits, where it was assumed that the function of the fundamental harmonic was dominant. Further, the piezoelectric harvesting structure can filter away most components higher than the first-order one due to its band-pass characteristics, so this approximation is feasible. In this case, the equivalent electrical impedance of the parallel SSHI circuit can be derived.

Here, the same methodology is adopted as follows. According to existing works [8], characteristic voltage and current waveforms of the parallel SSHI circuit are shown in Figure 7 . Then the output voltage of the harvesting structure can be described as follows:

$$
\begin{aligned}
& V_{p}(t) \\
& = \begin{cases}V_{\text {oc }}[1-\cos (\omega t)]-\gamma V_{\text {rec }}, & 0 \leq \omega t<\theta, \\
V_{\text {rec }}, & \theta \leq \omega t<\pi, \\
\gamma V_{\text {rec }}-V_{\text {oc }}[1+\cos (\omega t)], & \pi \leq \omega t<\pi+\theta, \\
-V_{\text {rec }}, & \pi+\theta \leq \omega t<2 \pi,\end{cases}
\end{aligned}
$$

where $V_{\text {rec }}$ is the rectified voltage, $V_{\text {oc }}$ is the open-circuit voltage amplitude, $\theta$ is the rectified blocked angle, and $\gamma$ is the voltage inversion factor defined as the voltage ratio of after and before the inversion action. Consider

$$
\gamma=-e^{-\pi /(2 Q)}
$$

where $Q$ is the quality factor of the switching loop. And the relation between $\theta$ and $V_{\text {rec }}$ can be formulated as follows based on (6):

$$
\cos \theta=1-\frac{(1+\gamma)\left(V_{\mathrm{rec}}+V_{D}\right)}{V_{\mathrm{oc}}},
$$




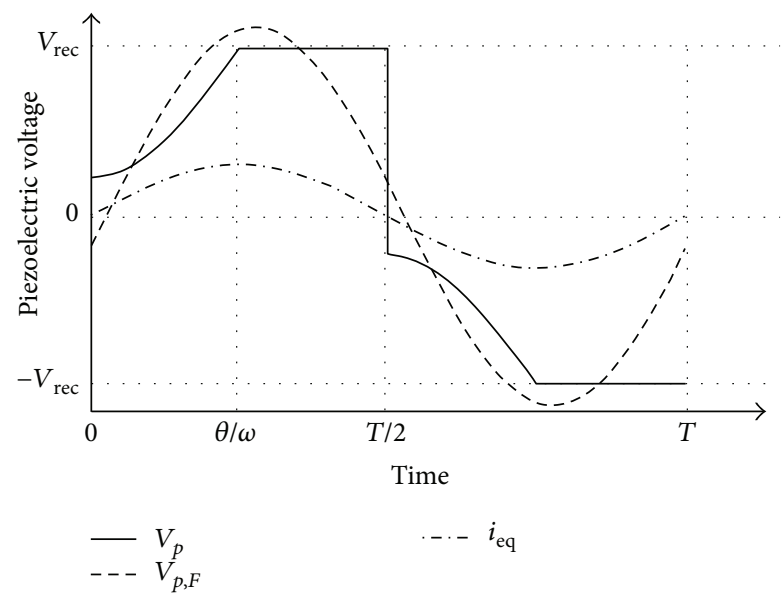

Figure 7: Characteristic voltage and current waveforms of the parallel SSHI circuit.

where $V_{D}$ is the forward voltage drop of the rectified diode. Finally, the equivalent load impedance is obtained as follows [14]:

$$
\begin{aligned}
& Z_{c}(j \omega)=\frac{1}{\pi \omega C_{p}}\left[(1-\cos \theta)\left(\frac{4}{1+\gamma}-1+\cos \theta\right)\right. \\
& +j(\sin \theta \cos \theta-\theta)] \triangleq \frac{1}{\pi \omega C_{p}}(\alpha+j \beta) .
\end{aligned}
$$

Next, it needs to calculate the equivalent electrical impedance of the parallel SSHI circuit. According to (10), the equivalent electrical impedance of the parallel SSHI circuit can be calculated as in (11):

$$
\begin{aligned}
& \frac{1}{Z_{\mathrm{P}-\mathrm{SSHI}}}=\frac{1}{Z_{c}}-\frac{1}{Z_{\mathrm{C}_{p}}}, \\
& Z_{\mathrm{P} \text {-SSHI }}=\frac{Z_{c} Z_{C_{p}}}{Z_{C_{p}}-Z_{c}}=\frac{\beta_{1} \pi+j\left(\beta_{1}^{2}+\beta_{2}^{2}+\beta_{2} \pi\right)}{\omega C_{p}\left[\beta_{1}^{2}+\left(\beta_{2}+\pi\right)^{2}\right]},
\end{aligned}
$$

where $\beta_{1}=(1-\cos \theta)(4 /(1+\gamma)-1+\cos \theta), \beta_{2}=\sin \theta \cos \theta-\theta$.

It can be seen from (11) that $Z_{\mathrm{P}-\mathrm{SSHI}}$ is a function of $\omega$, $\theta$, and $\gamma$. In addition, once the base excitation frequency and the structure of the parallel SSHI circuit are determined, $Z_{\text {P-SSHI }}$ will be a constant independent of the voltage source. Furthermore, $Z_{\mathrm{P}-\mathrm{SSHI}}$ is composed of real and imaginary components. The real component corresponds to a resistor and the imaginary component corresponds to an inductor or a capacitor according to the sign of $\beta_{1}^{2}+\beta_{2}^{2}+\beta_{2} \pi$. If $\beta_{1}^{2}+\beta_{2}^{2}+\beta_{2} \pi>0$, it will be an inductor. Otherwise, it will be a capacitor. Thus, the parallel SSHI circuit will be simplified to be a linear load, which includes a resistor in series with an inductor or a capacitor. Excitingly, a linear circuit can be easily built and analyzed by commercial FEA software. First of all, finite element model of the bimorph cantilever beam is built in the COMSOL software. Based on the value of $Z_{\text {P-SSHI }}$, then a resistor and a capacitor or inductor are added into "electrical part" as linear loads. Finally, the second-class

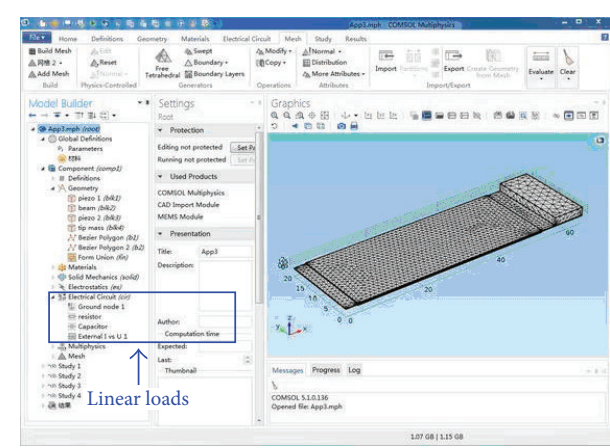

FIGURE 8: The second-class system-level coupled model of the PVEH system.

TABLE 1: Component parameters of the parallel SSHI circuit.

\begin{tabular}{lccc}
\hline$V_{D}$ & $C_{r}$ & $R$ & $R_{1}, R_{2}$ \\
\hline $0.5 \mathrm{~V}$ & $5 \mu \mathrm{F}$ & $100 \mathrm{k} \Omega$ & $200 \mathrm{k} \Omega$ \\
\hline
\end{tabular}

TABLE 2: Geometric dimensions of the bimorph cantilever beam.

\begin{tabular}{lc}
\hline Component & Dimensions \\
\hline Piezoelectric patch (PZT-5H) & $50 \mathrm{~mm} \times 20 \mathrm{~mm} \times 0.2 \mathrm{~mm}$ \\
Tip mass (steel) & $8 \mathrm{~mm} \times 20 \mathrm{~mm} \times 2 \mathrm{~mm}$ \\
Elastic base (copper) & $65 \mathrm{~mm} \times 20 \mathrm{~mm} \times 0.2 \mathrm{~mm}$ \\
\hline
\end{tabular}

coupled model can be built in the COMSOL software by connecting the above two parts, as shown in Figure 8.

\section{Simulation Validations}

6.1. Prototype of a PVEH System. In this section, a prototype of a PVEH system as in Figure 1 is studied. Basic component parameters of the parallel SSHI circuit shown in Figure 6 are listed in Table 1. Geometric dimensions and material properties of the bimorph cantilever beam are shown in Tables 2 and 3 , respectively. The vibration excitation is assumed to be $F=0.01 \sin 2 \pi f t$, where $f$ is the excitation frequency. In order to calculate the harvested power, a pure resistor $R=100 \mathrm{k} \Omega$ is selected as the load of the parallel SSHI circuit.

6.2. Comparison of the Two Classes of Coupled Models. For the first-class coupled model, the parameter identification method in Section 4 is used and equivalent model parameters of the harvesting structure are estimated as Table 4 by using the geometric and material properties in Tables 2 and 3.

Then the first-class coupled model of the PVEH system is built in the Multisim10.0, where the switch in the parallel SSHI circuit is realized by a self-powered electronic switch as shown in Figure 6. The harvested power from the first-class coupled model can be estimated as follows:

$$
P_{1}=\frac{V_{0}^{2}}{R}
$$

where $V_{0}$ is the output voltage across the load $R$. 
TABLE 3: Material properties of the bimorph cantilever beam.

\begin{tabular}{lcc}
\hline Materials & Properties & Values \\
\hline $\begin{array}{l}\text { Elastic } \\
\text { substrate }\end{array}$ & Density, $\rho_{s}\left(\mathrm{~kg} / \mathrm{m}^{3}\right)$ & 8500 \\
& Young's modulus, $E(\mathrm{Gpa})$ & 90 \\
\hline Tiezoelectric & Thickness, $h_{p}(\mathrm{~mm})$ & 0.2 \\
patch & Density, $\rho_{s}\left(\mathrm{~kg} / \mathrm{m}^{3}\right)$ & 7500 \\
& $\begin{array}{c}\text { Young's modulus, } E_{p}(\mathrm{Gpa}) \\
\text { Piezoelectric constant, }\end{array}$ & 66 \\
& $d_{31}(\mathrm{pm} / \mathrm{V})$ & -190 \\
& Permittivity, $\varepsilon_{33}^{s}(\mathrm{nF} / \mathrm{m})$ & 15.93 \\
\hline
\end{tabular}

TABLE 4: Equivalent model parameters of the harvesting structure.

\begin{tabular}{lcccc}
\hline$V_{\text {eq }}$ & $R_{\text {eq }}$ & $L_{\text {eq }}$ & $C_{\text {eq }}$ & $C_{p}$ \\
\hline $22.4 \mathrm{~V}$ & $170.2 \mathrm{k} \Omega$ & $17.18 \mathrm{kH}$ & $2.09 \mathrm{nF}$ & $66.34 \mathrm{nF}$ \\
\hline
\end{tabular}

For the second-class coupled model, the equivalent impedance parameters of the parallel SSHI circuit are calculated based on (11). It can be seen that the electrical impedance of the parallel SSHI circuit is a linear load involving a resistor in series with a capacitor. Then the second-class coupled model of the PVEH system is built in COMSOL4.2a. By using FEA, the current $I_{p}$ flowing into the equivalent impedance $Z_{\mathrm{P}-\mathrm{SSHI}}$ and the voltage $V_{p}$ (e.g., $V_{0}$ ) across the equivalent impedance $Z_{\mathrm{P}-\mathrm{SSHI}}$ can be calculated, respectively. By this way, the harvested power from the second-class coupled model can be estimated as follows:

$$
P_{2}=\frac{V_{p} I_{p} \cos \varphi}{2},
$$

where the angle $\varphi$ can be calculated as follows:

$$
\varphi=\tan ^{-1}\left[\frac{\operatorname{Im}\left(Z_{\text {P-SSHI }}\right)}{\operatorname{Re}\left(Z_{\text {P-SSHI }}\right)}\right] .
$$

In addition, the theoretic harvested power can be described as follows:

$$
P_{3}=\frac{V_{\mathrm{eq}}^{2} \operatorname{Re}\left[Z_{c}(j \omega)\right]}{2\left|R_{\mathrm{eq}}+j\left(\omega_{1} L_{\mathrm{eq}}-1 / \omega_{1} C_{\mathrm{eq}}\right)+Z_{c}(j \omega)\right|^{2}} .
$$

In order to compare the two classes of coupled models, the same excitation is used. Here the excitation frequency is set to $f=27 \mathrm{~Hz}$ and its amplitude is equal to estimated $V_{\text {eq. }}$. Finally, harvested powers from the two classes of coupled models under different $R$ are estimated as shown in Figure 9. Furthermore, frequency responses of the harvested power of the two coupled models are shown in Figure 10.

It can be seen from Figures 9 and 10 that harvested powers from the two classes of coupled models are almost the same under different loads and excitation frequencies. In addition, both estimated harvested powers are very close to the theoretic values. Thus the proposed coupled models can be used for system-level optimizations in engineering applications.

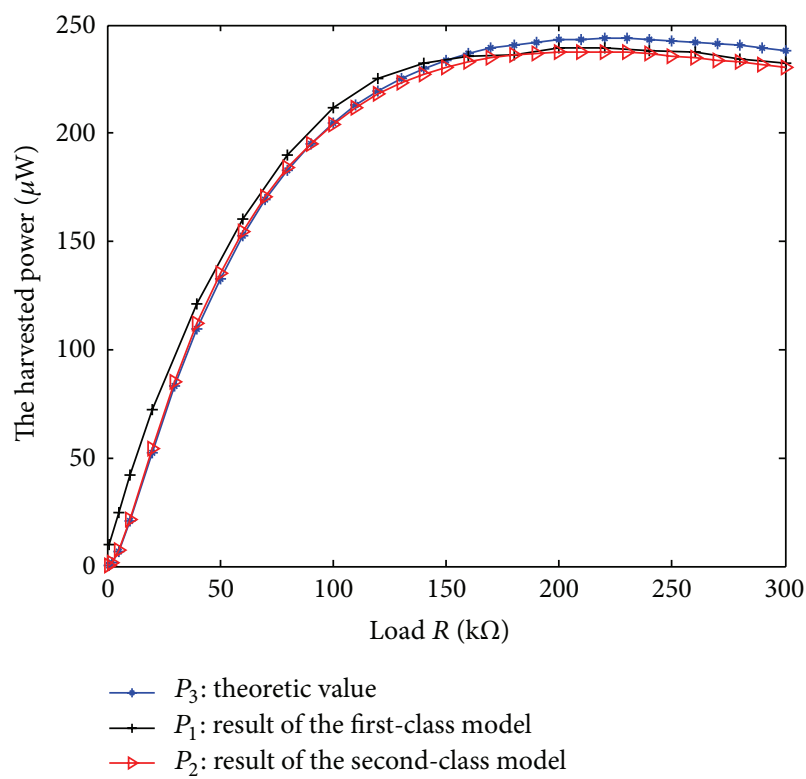

FIGURE 9: Comparison of harvested powers from the two classes of coupled models.

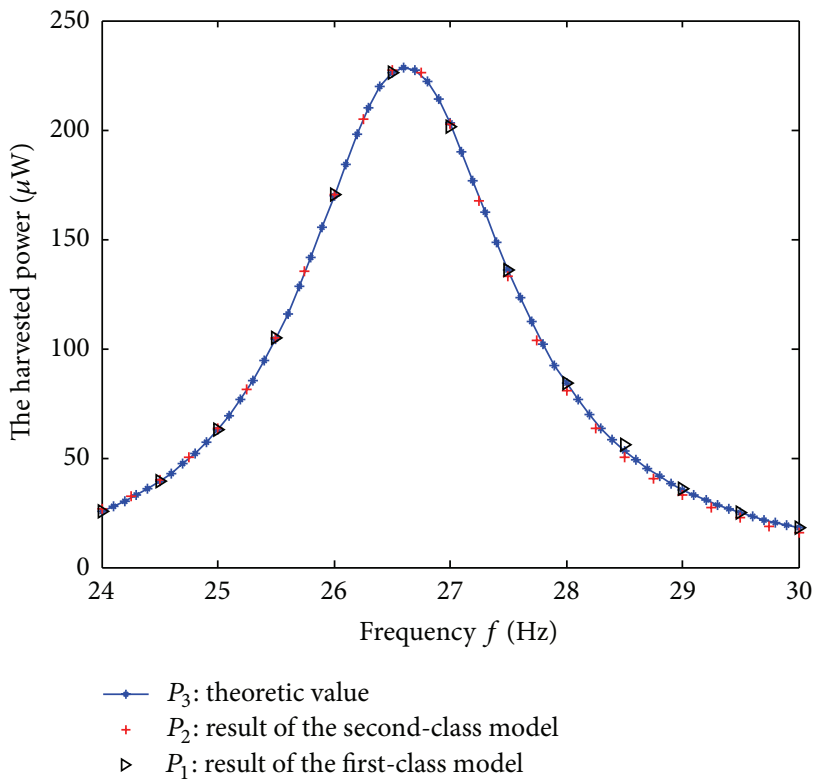

FIGURE 10: Comparison of frequency responses of harvested power from the two classes of coupled models.

6.3. Parameters Optimization of the PVEH System Using System-Level Coupled Models. As we all know, the two capacitors $\left(C_{1}=C_{2}=C\right)$ and the inductor $(L)$ in the parallel SSHI circuit (shown in Figure 6) are key parameters. Thus, they should be optimized to obtain the highest overall efficiency of a PVEH system under a given vibration excitation. Here a harvesting structure described as in Tables 1 and 2 is adopted and fixed. The excitation is assumed as $F_{1}=0.01 \sin 40 \pi t$. Then the first-class coupled model is used to optimize $C$ and $L$ instantaneously. Finally, the 3D plot of the harvested power with $C$ and $L$ is shown in Figure 11. 
Shock and Vibration

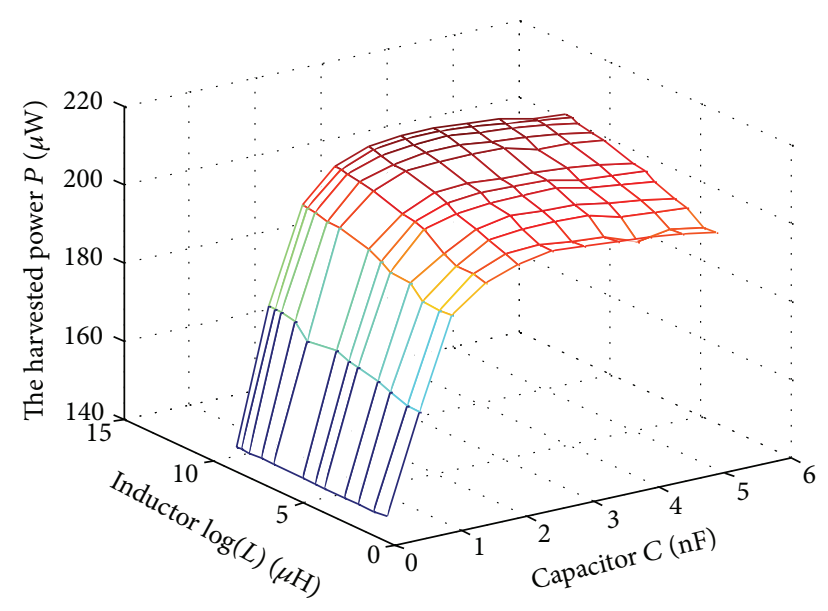

Figure 11: 3D plot of the harvested power with $C$ and $L$.

It can be seen from Figure 11 that the maximum harvested power is achieved when $C=3 \mathrm{nF}$ and $L=10 \mathrm{mH}$. In addition, the maximum harvested power increases very little when $L$ is larger than $10 \mathrm{mH}$. Similarly, the secondclass coupled model can be used to optimize geometric and material properties of the harvesting structure, which will not be repeated in this paper.

\section{Conclusions}

Due to coupling interactions between energy harvesting structure and the interface circuit in a practical PVEH system, it is much necessary to build a system-level coupled model for optimizing the PVEH system and improving its overall conversion efficiency. In this paper, two classes of systemlevel coupled models are proposed based on joint finite element and circuit analysis.

Main conclusions of this paper may include the following: (i) Both coupled models lead to similar results, so either one can be used for system-level optimizations of PVEH systems in engineering applications. (ii) The system-level circuit model is based on lumped-parameter model for the harvesting structure, so that geometric dimensions cannot be involved and optimized, while the interface circuit can be optimized easily. (iii) The system-level finite element model is based on equivalent impedance of the interface circuit, so it is inconvenient to optimize the interface circuit. While geometric dimensions of the harvesting structure can be involved easily, (iv) equivalent circuit model parameters of the harvesting structure can be estimated by FEA, instead of experimental measurements. However, this method may be unfit for irregular piezoelectric cantilever beams. (v) The highest overall efficiency of a PVEH system would be achieved by using the proposed coupled models. In addition, it must be addressed that approximations are adopted in both coupled models, so certain deviations may exist in the results. Thus, experiments should be performed to testify the proposed two system-level coupled models in the future.

\section{Conflict of Interests}

The authors declare that there is no conflict of interests regarding the publication of this paper.

\section{Acknowledgment}

This work was supported by the National Natural Science Foundation of China (Grant nos. 51275520 and 51577189).

\section{References}

[1] L. G. W. Tvedt, D. S. Nguyen, and E. Halvorsen, "Nonlinear behavior of an electrostatic energy harvester under wideand narrowband excitation," Journal of Microelectromechanical Systems, vol. 19, no. 2, Article ID 5404427, pp. 305-316, 2010.

[2] A. Rahimi, Ö. Zorlu, A. Muhtaroǧlu, and H. Külah, "Fully selfpowered electromagnetic energy harvesting system with highly efficient dual rail output," IEEE Sensors Journal, vol. 12, no. 6, pp. 2287-2298, 2012.

[3] J. W. Kan, J. H. Qiu, K. H. Tang, K. J. Zhu, and C. H. Shao, "Modeling and simulation of piezoelectric composite diaphragms for energy harvesting," International Journal of Applied Electromagnetics and Mechanics, vol. 30, no. 1-2, pp. 95106, 2009.

[4] Z. S. Chen, B. Guo, C. C. Cheng, H. W. Shi, and Y. M. Yang, "Chaotic dynamics-based analysis of broadband piezoelectric vibration energy harvesting enhanced by using nonlinearity," Shock and Vibration, vol. 2016, Article ID 3584740, 11 pages, 2016.

[5] R. Caliò, U. B. Rongala, D. Camboni et al., "Piezoelectric energy harvesting solutions," Sensors, vol. 14, no. 3, pp. 4755-4790, 2014.

[6] N. Kong, D. S. Ha, A. Erturk, and D. J. Inman, "Resistive impedance matching circuit for piezoelectric energy harvesting," Journal of Intelligent Material Systems and Structures, vol. 21, no. 13, pp. 1293-1302, 2010.

[7] E. Lefeuvre, A. Badel, C. Richard, and D. Guyomar, "Piezoelectric energy harvesting device optimization by synchronous electric charge extraction," Journal of Intelligent Material Systems and Structures, vol. 16, no. 10, pp. 865-876, 2005.

[8] D. Guyomar, A. Badel, E. Lefeuvre, and C. Richard, "Toward energy harvesting using active materials and conversion improvement by nonlinear processing," IEEE Transactions on Ultrasonics, Ferroelectrics, and Frequency Control, vol. 52, no. 4, pp. 584-594, 2005.

[9] L. L. Silva, M. A. Savi, P. C. Monteiro, and T. A. Netto, "On the nonlinear behavior of the piezoelectric coupling on vibrationbased energy harvesters," Shock and Vibration, vol. 2015, Article ID 739381, 15 pages, 2015.

[10] A. Romani, R. P. Paganelli, E. Sangiorgi, and M. Tartagni, "Joint modeling of piezoelectric transducers and power conversion circuits for energy harvesting applications," IEEE Sensors Journal, vol. 13, no. 3, pp. 916-925, 2013.

[11] R. P. Paganelli, A. Romani, A. Golfarelli, M. Magi, E. Sangiorgi, and M. Tartagni, "Modeling and characterization of piezoelectric transducers by means of scattering parameters. Part I: theory," Sensors and Actuators, A: Physical, vol. 160, no. 1-2, pp. 9-18, 2010.

[12] Y. T. Hu, T. Hu, and Q. Jiang, "Coupled analysis for the harvesting structure and the modulating circuit in a piezoelectric bimorph energy harvester," Acta Mechanica Solida Sinica, vol. 20, no. 4, pp. 296-308, 2007. 
[13] N. G. Elvin and A. A. Elvin, "A coupled finite element-circuit simulation model for analyzing piezoelectric energy generators," Journal of Intelligent Material Systems and Structures, vol. 20, no. 5, pp. 587-595, 2009.

[14] Y. C. Shu and I. C. Lien, "Analysis of power output for piezoelectric energy harvesting systems," Smart Structures and Materials, vol. 15, no. 3, pp. 1499-1512, 2006.

[15] J. R. Liang and W.-H. Liao, "Impedance modeling and analysis for piezoelectric energy harvesting systems," IEEE/ASME Transactions on Mechatronics, vol. 17, no. 6, pp. 1145-1157, 2012.

[16] J. R. Liang and W.-H. Liao, "Improved design and analysis of self-powered synchronized switch interface circuit for piezoelectric energy harvesting systems," IEEE Transactions on Industrial Electronics, vol. 59, no. 4, pp. 1950-1960, 2012. 


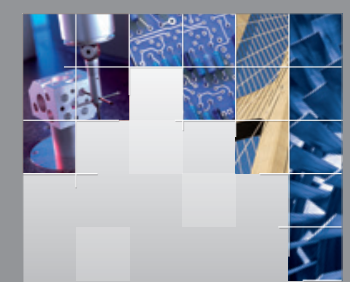

\section{Enfincering}
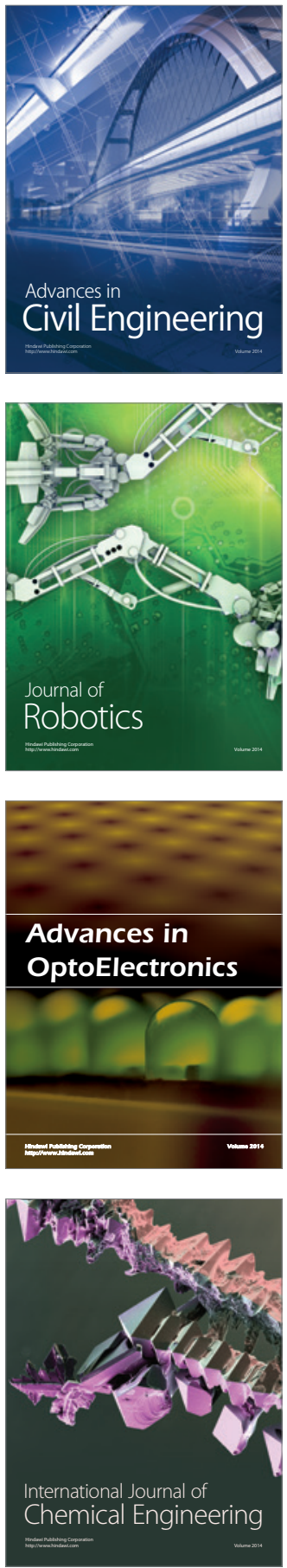

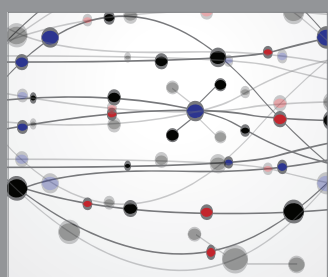

The Scientific World Journal

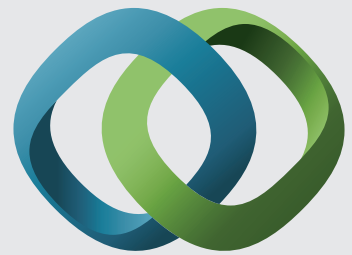

\section{Hindawi}

Submit your manuscripts at

http://www.hindawi.com
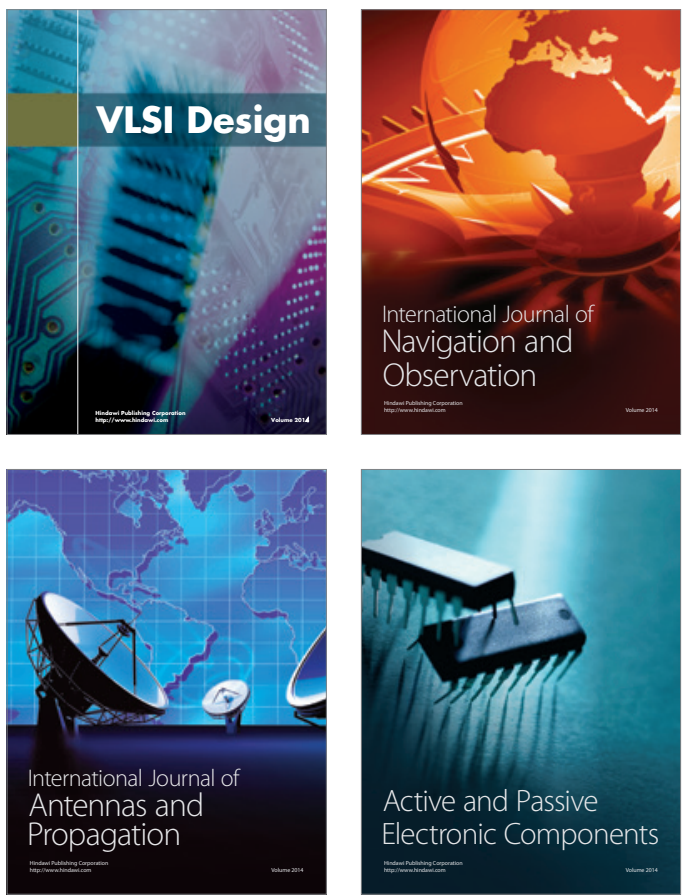
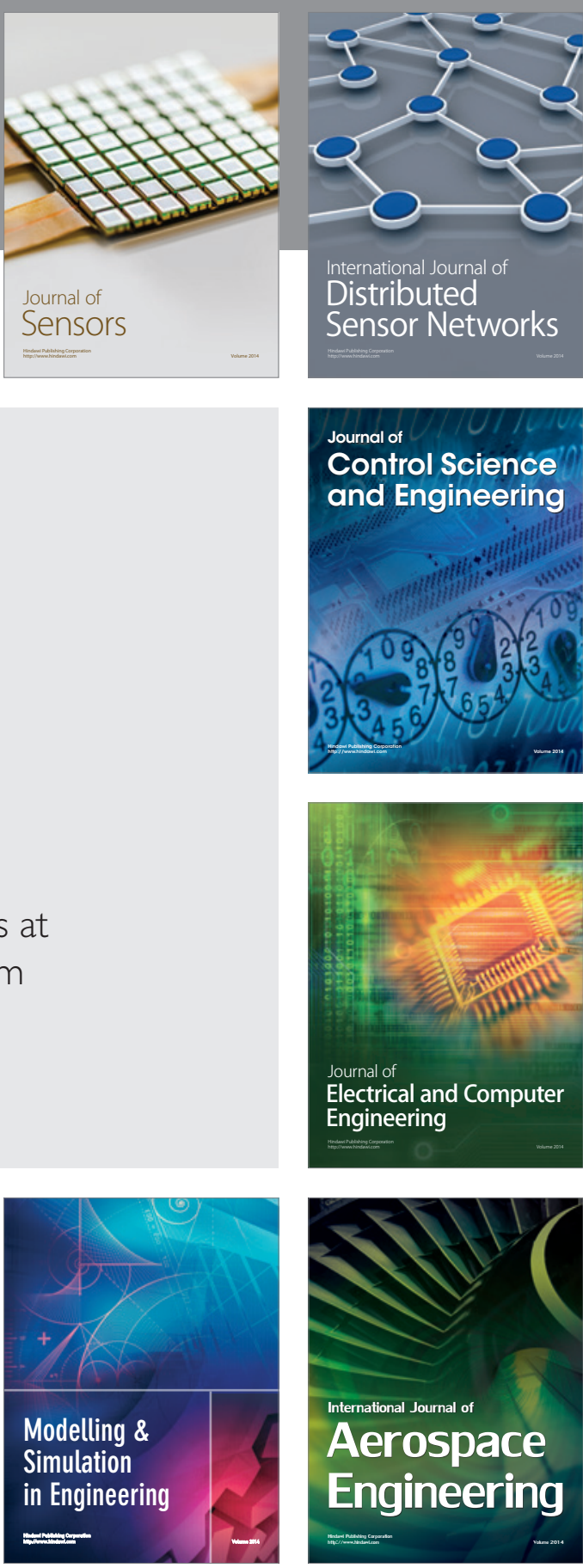

International Journal of

Distributed

Sensor Networks

Journal of

Control Science

and Engineering
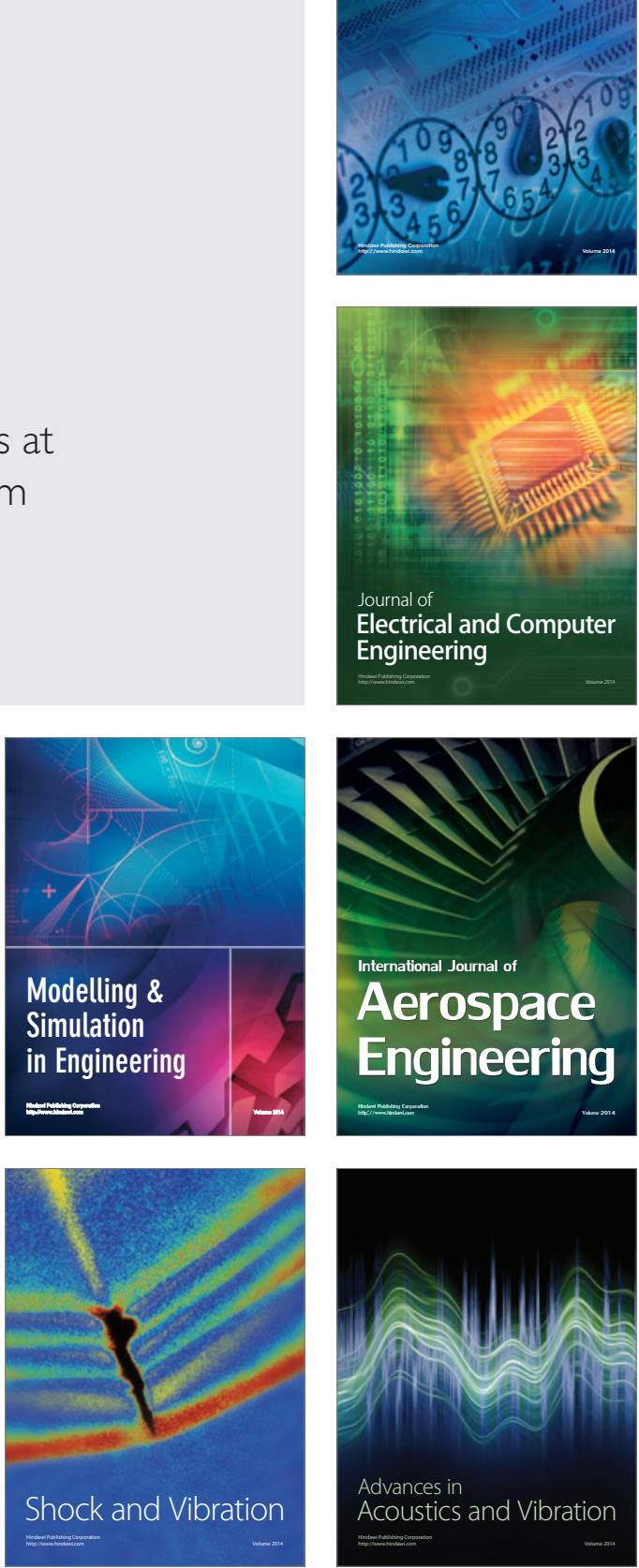